\title{
Assessing resting energy expenditure in overweight and obese adolescents in a clinical setting: validity of a handheld indirect calorimeter
}

\author{
Paula Woo', Gayathri Murthy ${ }^{2,3}$, Cindy Wong ${ }^{2,3}$, Brenden Hursh ${ }^{1,3}$, Jean-Pierre Chanoine ${ }^{1,3}$ and Rajavel Elango ${ }^{2,3,4}$
}

BACKGROUND: Accurately determining energy requirements is key for nutritional management of pediatric obesity. Recently, a portable handheld indirect calorimeter, MedGem (MG) has become available to measure resting energy expenditure (REE). Our work aims to determine the clinical validity and usefulness of MG to measure REE in overweight and obese adolescents.

METHODS: Thirty-nine overweight and obese adolescents (16 male (M): 23 female (F), 15.2 $\pm 1.9 \mathrm{y}$, BMI percentile: $98.6 \pm 2.2 \%)$ and 15 normal weight adolescents (7M: 8F, age $15.2 \pm 2.0$ y, BMI percentile: $39.2 \pm 20.9 \%$ ) participated. REE was measured with both $M G$ and standard indirect calorimeter (VMax) in random order.

RESULTS: MG REE $(1,600 \pm 372 \mathrm{kcal} / \mathrm{d})$ was lower than VMax $\operatorname{REE}(1,727 \pm 327 \mathrm{kcal} /)$ in the overweight and obese adolescents. Bland Altman analysis (MG -VMax) showed a mean bias of $-127 \mathrm{kcal} / \mathrm{d}(95 \% \mathrm{Cl}=-72$ to $-182 \mathrm{kcal} / \mathrm{d}, P<0.001)$, and a proportional bias existed such that lower measured REE by VMax was underestimated by MG, and higher measured REE by VMax were overestimated by MG.

CONCLUSION: MG systematically underestimates REE in the overweight and adolescent population, thus the MG portable indirect calorimeter is not recommended for routine use. Considering that it is a systematic underestimation of REE, MG may be clinically acceptable, only if used with caution.

Chor hildhood obesity is a major public health issue around the world and has a huge impact on the health of millions of young people. In Canada, nearly one out of three children and youth are overweight or obese (1). Energy balance is an important physiological aspect of obesity and sustainable weight loss may be achieved by creating a negative energy balance. To achieve an appropriate weight for age, the Canadian Obesity Guidelines suggests that a negative energy balance be achieved by a well-balanced, calorie-reduced diet and increased physical activity (2). The Academy of Nutrition and Dietetics recommends the use of indirect calorimeters to determine caloric targets for weight loss in obese youth (3). Both of these recent guidelines acknowledge the importance of understanding or measuring accurate energy requirements as part of a comprehensive pediatric nutrition assessment for weight loss in this population.

Resting energy expenditure (REE) represents $60-75 \%$ of daily total energy expenditure (4), and can be quantified using traditional open-circuit indirect calorimeter carts or metabolic carts and is primarily based on measurement of oxygen consumption $\left(\mathrm{VO}_{2}\right)$ and carbon dioxide $\left(\mathrm{VCO}_{2}\right)(5,6)$. This method has long been established as the reference standard for measuring REE $(7,8)$. However, metabolic carts are expensive, require careful calibration, are less mobile and require longer testing time $(9,10)$.

Portable handheld devices such as the MedGem (MG), have become available as an alternative to the traditional calorimeter (11). MG measures $\mathrm{VO}_{2}$ and calculates VCO2, based on an assumed constant respiratory quotient (RQ) of 0.85 , where RQ is usually derived as a ratio of $\mathrm{VCO} 2 / \mathrm{VO} 2$. The validity of the portable indirect calorimeter has been assessed in adults, both healthy (12-14) and unhealthy (e.g., eating disorders, cancer) (15-19) with varying results. In obese adults, earlier studies have reported that the portable indirect calorimeters overestimate REE, when compared to the standard indirect calorimeters compared (20-22). In healthy normal weight children, Fields et al. (23) showed that hand-held calorimeters overestimate REE, while Nieman et al. (24) showed that the handheld calorimeter provides reliable REE measures compared to a Douglas bag method, a measure of pulmonary ventilation and respiratory gas exchange that provides an estimation of energy expenditure. Whether the portable indirect calorimeters are reliable and valid to use in an adolescent overweight and obese population is unknown. Our goal was to validate the use of MG in our pediatric Endocrinology Clinic and Type 2 Diabetes clinic. Thus, the primary objective in the current study is to compare measured REE from the handheld indirect calorimeter (MG) against a standard indirect calorimeter (VMax) in overweight and obese adolescents. Because energy

\footnotetext{
The first two authors shared first authorship.

'Division of Endocrinology, BC Children's Hospital, Vancouver, British Columbia, Canada; ${ }^{2}$ Research Institute, BC Children's Hospital, Vancouver, British Columbia, Canada; ${ }^{3}$ Department of Pediatrics, University of British Columbia, Vancouver, British Columbia, Canada; ${ }^{4}$ School of Population and Public Health, University of British Columbia, Vancouver, British Columbia, Canada. Correspondence: Rajavel Elango (relango@cfri.ubc.ca)

Received 28 January 2016; accepted 2 August 2016; advance online publication 19 October 2016. doi:10.1038/pr.2016.182
} 
requirements are often estimated using predictive equations (25-27) in a clinical setting, our secondary objective is to compare measured REE against predicted REE in the overweight and obese adolescents. We hypothesized that the measured REE will be comparable between the portable and standard indirect calorimeters, and that the predicted REE using equations will not be comparable to the standard indirect calorimeter measured REE.

\section{RESULTS}

\section{Participant Characteristics}

Forty-one overweight, and 15 normal weight (BMI between 15 th and 85 th percentile) children were recruited. Because two overweight participants had difficulties breathing in the supine position and their data did not reach steady state, data are presented for 39 overweight children (23 female (F):16 male (M)) and 15 normal-weight (8F:7M) children (Table 1). Weight and $\mathrm{BMI}$ (mean $\pm \mathrm{SD})$ were significantly higher $(P<0.001)$ in the overweight group $\left(88.1 \pm 20.2 \mathrm{~kg}, 32.3 \pm 5.8 \mathrm{~kg} / \mathrm{m}^{2}\right)$ than in the normal-weight group $\left(51.3 \pm 5.8 \mathrm{~kg}, 19.4 \pm 1.9 \mathrm{~kg} / \mathrm{m}^{2}\right)$. Mean BMI of all children in the overweight group was $\geq 99$ th percentile for age and gender. Body fat percentage was significantly higher in the overweight children when compared to the normal-weight children $(46.5 \pm 4.5$ vs. $28.3 \pm 7.1 \%$; $P<$ 0.001 . Fat-free mass was also significantly higher in the overweight children when compared to the normal-weight children $(48.1 \pm 7.5$ vs. $36.8 \pm 6.3 \mathrm{~kg} ; P<0.001$.

\section{REE Measurement by VMax and MG}

On average, REE measured by MG was $\sim 8 \%$ lower when compared to REE measured by VMax $(1,600 \pm 404$ vs. $1,727 \pm 349$ $\mathrm{kcal} / \mathrm{d}, P<0.001$ ) in the overweight group (Table 2). Similarly, on average, REE measured by MG was $~ 16 \%$ lower than REE measured by VMax $(1,181 \pm 230$ vs. $1,370 \pm 234 \mathrm{kcal} / \mathrm{d}$, $P<0.001)$ in the normal-weight group. Twenty-nine of the 39 children (74\%) had REE measured by MG within the clinically acceptable a priori set at $\pm 250 \mathrm{kcal} / \mathrm{d}$. This is based on the calculation that a $250 \mathrm{kcal} / \mathrm{d}$ change in energy intake would lead to a half-pound weight loss in a week, as previously applied by Curtin et al. (28), which is in line with recommendations by the American Academy of Pediatrics for treatment of and overweight and obese adolescents (29).

Table 1. Subject characteristics ${ }^{\mathrm{a}}$

\begin{tabular}{lcc}
\hline Variable & Overweight $(n=39)$ & Normal-weight $(n=15)$ \\
\hline Age (years) & $15.2 \pm 1.8(12.4-18.8)$ & $15.5 \pm 2.0(12.6-18.4)$ \\
Sex $(\mathrm{F} / \mathrm{M})$ & $23 / 16$ & $8 / 7$ \\
Height $(\mathrm{cm})$ & $164.4 \pm 8.7(141-189.3)$ & $162.2 \pm 6.9(152.3-174.5)$ \\
Weight $(\mathrm{kg})$ & $88.1 \pm 20.2^{*}(55.5-147.9)$ & $51.3 \pm 5.8(41.7-66.2)$ \\
BMl $\left(\mathrm{kg} / \mathrm{m}^{2}\right)$ & $32.4 \pm 5.8^{*}(23.4-53.7)$ & $19.4 \pm 1.9(15.8-22.4)$ \\
Fat-free mass $(\mathrm{kg})$ & $46.6 \pm 8.9(30.2-71.3)$ & $36.8 \pm 6.3(29.3-52.9)$ \\
Body fat $(\%)$ & $46.1 \pm 4.5^{*}(36.4-57.1)$ & $28.3 \pm 7.1(18.6-38.1)$ \\
\hline avalues are mean \pm SD (range). ${ }^{*}$ Significantly $(P<0.001)$ & different compared to normal- \\
weight group. &
\end{tabular}

\section{Correlation Analysis}

Pearson correlation coefficient $(r=0.91, P<0.001,95 \% \mathrm{CI}=$ 0.83-0.95) showed a strong linear relationship between REE measured using MG and VMax in the overweight group (Figure 1). There was also a strong linear relationship $(r=0.83$, $P<0.001,95 \% \mathrm{CI}=0.54,0.94)$ between REE measured using MG and VMax in the normal-weight group (Figure 2).

\section{Bland Altman Analysis}

Mean overall difference in values obtained with the two different methods (bias) and limits of agreement (95\% confidence limits for the bias) were determined. In the overweight children, there was a mean bias of $-127 \pm 168 \mathrm{kcal} / \mathrm{d}$ between measured REE using MG when compared to REE measured using VMax (Figure 3). The upper and lower 95\% limits of agreement were 203 and $-457 \mathrm{kcal} / \mathrm{d}$. In addition to the fixed systematic bias, there was a proportional bias in the measured REE differences between the MG and VMax in the overweight group, such that lower measured REE by VMax was underestimated by MG, and higher measured REE by VMax were overestimated by MG. In the normal-weight children, there was a mean bias of $-190 \pm 136 \mathrm{kcal} / \mathrm{d}$ between measured REE using MG and VMax (Figure 4). The upper and lower 95\% limits of agreement were 79 and $-458 \mathrm{kcal} / \mathrm{d}$. There was no

Table 2. Measured resting energy expenditure (REE) and $\mathrm{VO}_{2}$ using MedGem (MG) and standard indirect calorimeter (VMax) ${ }^{\mathrm{a}, \mathrm{b}}$

\begin{tabular}{lcc}
\hline & Overweight $(n=39)$ & Normal-weight $(n=15)$ \\
\hline & $1,727 \pm 349$ & REE $(\mathrm{kcal} / \mathrm{d})$ \\
VMax & $1,600 \pm 404^{*}$ & $1,370 \pm 234$ \\
MedGem (MG) & $\mathrm{VO}_{2}(\mathrm{ml} / \mathrm{min})$ \\
VMax & $245 \pm 50$ & $194 \pm 230^{*}$ \\
MedGem (MG) & $231 \pm 58$ & $170 \pm 31$ \\
\hline
\end{tabular}

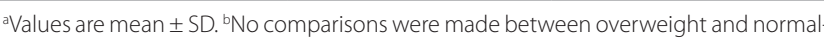
weight group. *Significantly $(P<0.001)$ different when compared to REE from VMax.

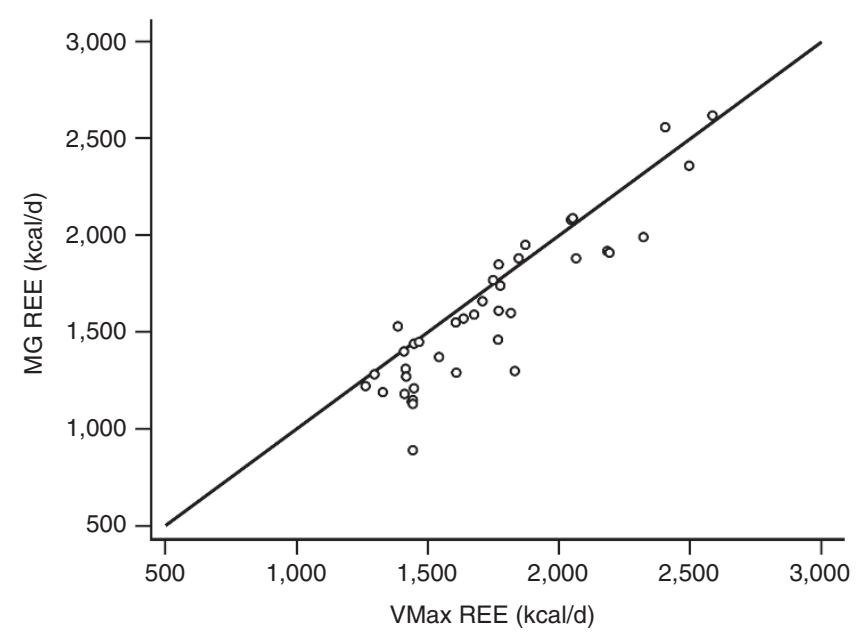

Figure 1. Correlation between MG and VMax resting energy expenditure (REE) measurements in the overweight group. Pearson $r=0.91,{ }^{*} P<0.001$, $n=39$. 


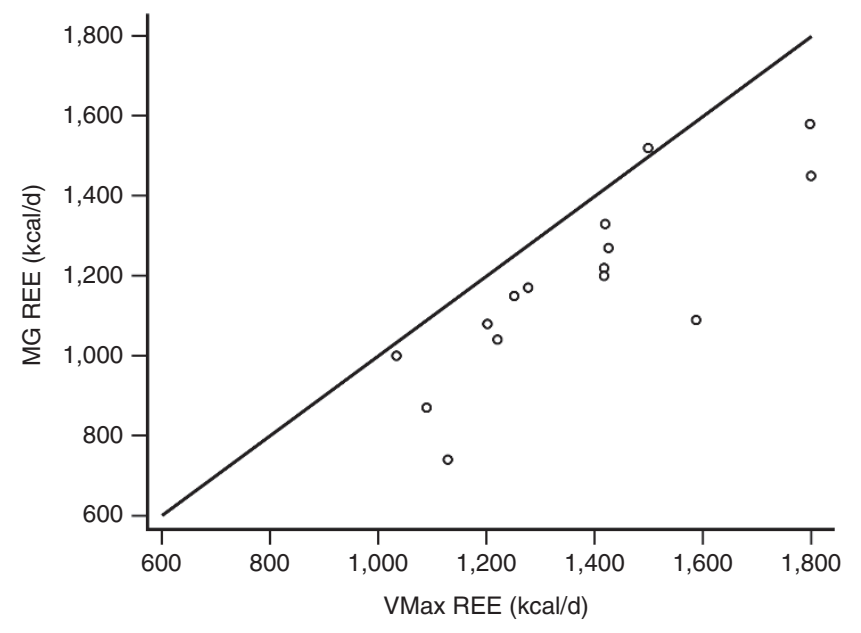

Figure 2. Correlation between MG and VMax resting energy expenditure (REE) measurements in the normal-weight group. Pearson $r=0.83,{ }^{*} P<$ $0.001, n=15$.

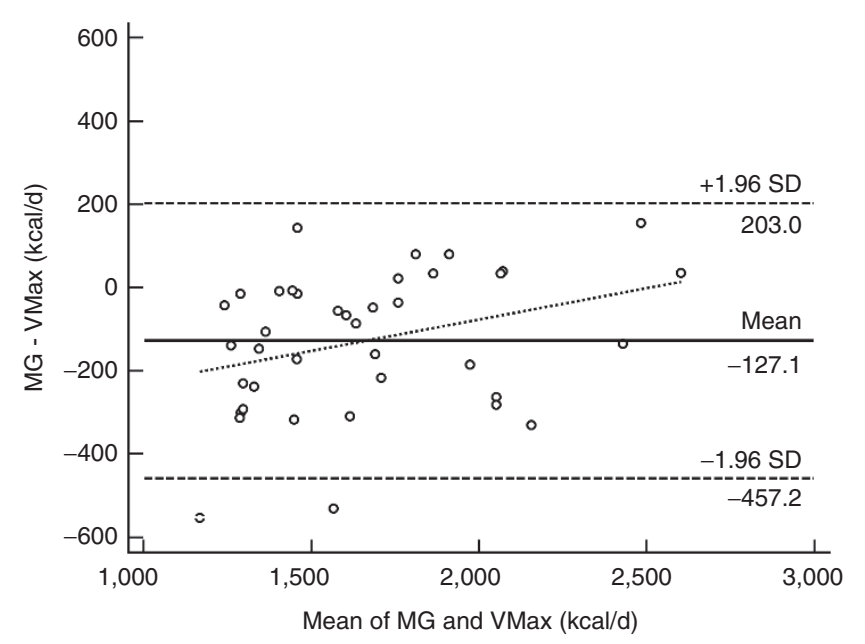

Figure 3. Bland-Altman plot showing the mean bias and limits of agreement between MG and VMax in overweight group. The solid line represents mean bias $(-127.1 \mathrm{kcal} / \mathrm{d}$, a systematic underestimation in MG resting energy expenditure (REE)) and dashed lines represent \pm 2 SD (limits of agreement). The dotted line represents the linear trend of the differences between the two measures depicting proportional bias $\left({ }^{*} P<\right.$ $0.042, n=39$ ).

proportional bias observed in the measured REE by MG in the normal-weight children.

\section{Comparison of Measure REE Using MG Against Predictive Equations}

In the overweight/obese group, predictive equations overestimate REE by $\sim 30 \%$ (Table 3 ). The Molnar equation had the least mean bias of $2 \%$ when compared with the metabolic cart (VMax) REE predicted using the WHO/FAO/UNU equation showed an overestimation by up to $41 \%$.

\section{DISCUSSION}

Results from our study indicate that the handheld indirect calorimeter (MG) significantly underestimates REE (mean bias, which represents a systematic error, of $-127 \mathrm{kcal} / \mathrm{d}$ ) when

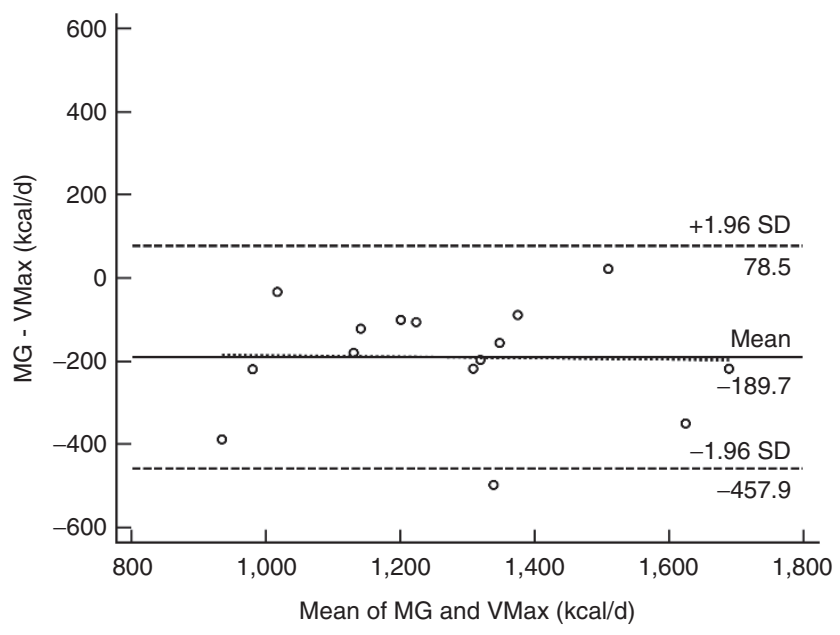

Figure 4. Bland-Altman plot showing the mean bias and limits of agreement between MG and VMax in normal-weight group. The solid line represents mean bias $(-189.7 \mathrm{kcal} / \mathrm{d}$, a systematic underestimation in MG resting energy expenditure (REE)) and dashed lines represent \pm 2 SD (limits of agreement). The dotted line represents the linear trend of the differences between the two measures depicting no proportional bias ( $P$ $=0.923, n=15$ ).

Table 3. Predicted resting energy expenditure (REE) compared to measured resting energy expenditure ${ }^{a}$

\begin{tabular}{|c|c|c|}
\hline Predictive equation & Overweight $(n=39)$ & Normal-weight $(n=15)$ \\
\hline & \multicolumn{2}{|c|}{$\operatorname{REE}(\mathrm{kcal} / \mathrm{d})$} \\
\hline Measured REE (VMax) & $1,727 \pm 349$ & $1,370 \pm 234$ \\
\hline Measured REE (MG) & $1,600 \pm 404$ & $1,181 \pm 230$ \\
\hline Harris Benedict & $1,741 \pm 248$ & $1,438 \pm 116^{*}$ \\
\hline WHO/FAO/UNU & $1,890 \pm 359^{*}$ & $1,459 \pm 150^{*}$ \\
\hline Molnar & $1,758 \pm 325^{* *}$ & $\mathrm{n} / \mathrm{a}$ \\
\hline
\end{tabular}

compared to the standard indirect calorimeter (VMax), in an adolescent overweight population. A proportional bias also existed where the underestimation decreased with a higher measured REE. The limits of agreement, which reflects random error and represent the range of values in which the agreement between methods will lie for $95 \%$ of the sample, were +203 to $-457 \mathrm{kcal} / \mathrm{d}$. These results suggest that the MG is not universally acceptable for routine use in the pediatric weight management practice. Considering that the systematic effect is an underestimate, if the MG is to be used in clinical settings for overweight and adolescent weight management, caution needs to be exercised. Frequent monitoring of the caloric recommendation should be adopted for clinic patients.

To the best of our knowledge, our study is the first to compare MG to a standard indirect calorimeter in an adolescent overweight population and adds to the current body of related literature, where MG was tested against traditional indirect calorimetry or predictive equations in other populations. Earlier studies show conflicting results regarding REE comparisons between the portable and standard indirect calorimeters: 


\section{Articles | wooetal.}

in a larger sample of nonobese children $(n=100), 10-13 \mathrm{y}$, Fields et al. (23) found MG to overestimate REE by $8 \%$ when compared with a traditional indirect calorimeter. On the other hand, two studies in adults reported that MG underestimated REE in stable patients on home nutrition support (17) and patients with cancer (19). In the study with patients on home nutrition support, a difference of $\sim 130 \mathrm{kcal} / \mathrm{d}$ between measurements of MedGem and a standard indirect calorimeter with limits of agreement beyond the clinically acceptable \pm 250 $\mathrm{kcal} / \mathrm{d}$ was observed (17). In the study with cancer patients (19), where the clinical acceptability was a priori set at $<5 \%$ difference, less than half of the patients had measured REE by the hand held device within clinically acceptable limits. Thus, in general the MG measured REE in most populations does not compare well with the standard indirect calorimeter. One of the reasons for this lack of comparison could be due to the fact that the MG assumes a constant RQ of 0.85 , and measures only $\mathrm{VO}_{2}(11)$. In the present study, a lower measured $\mathrm{VO}_{2}$, and an assumed RQ of 0.85 used to calculate $\mathrm{VCO}_{2}$ combined to underestimate the REE consistently in both the overweight and obese children, and the healthy children.

REE is an essential component of total energy expenditure as it represents up to $75 \%$ of the total energy needs. Thus, a reasonable measure of REE is important to estimate energy intake goals for weight management in overweight/obese individuals. Although REE can be determined accurately using the traditional indirect calorimeters, its usage is limited due to the cost, maintenance and the expertise required in operating the device (5). Therefore in a clinical setting, energy requirements are estimated using predictive equations. Many studies have validated a variety of equations to estimate resting energy needs in the overweight adolescent population, although most equations are derived from a healthy weight cohort of children (26). Thus, predictive equations derived from normal weight populations may overestimate REE up to $25 \%$ in some cases $(5,30)$ in overweight and obese population. Body weights can be $20 \%$ or more than the ideal weight in obese individuals, and can introduce an additional value in the estimation of caloric needs. Although some studies have suggested the use of ideal body weight or adjusted body weight (31), it is controversial and not widely followed. Among the predictive equations the Molnar equation (27) and Harris Benedict equation (32) compared reasonably well in the present study, and the FAO/WHO/ UNU (33) equation provided significantly higher REE values in the obese and overweight population. The Molnar equation was developed in an adolescent population (27), and thus provides a reasonable alternative when directly measuring REE is not an option. Our results are in agreement with Hofsteenge et al. (26) who found that the Molnar equation most accurately predicted REE when compared to measured REE by standard indirect calorimeter in 121 overweight and obese adolescents. Thus, while it is accepted that the standard indirect calorimeter is still the preferred method to estimate REE in adolescents, in clinical practice where the measurement is not available, the Molnar equation seems to predict REE accurately to make diet prescriptions.
Although the MG is likely to underestimate REE and has limits of agreement beyond a priori set at $\pm 250 \mathrm{kcal} / \mathrm{d}$ in our findings, majority of the children (74\%) were within the \pm 250 $\mathrm{kcal} / \mathrm{d}$. In addition, the MG can offer an objective measure of $\mathrm{REE}$ as a starting caloric goal in diet prescription. There is reasonable evidence that providing empowerment to vulnerable teenagers, such as obese/overweight children in our study with an objective measure of a caloric goal based on a personalized test, can provide additional motivation to make small changes in their dietary intake (34). These small changes in nutrition (energy intake) in addition to physical activity behaviors can prevent weight gain (35). Thus, an individually measured REE provides an additional tool to aid in the weight management strategy, as shown earlier in overweight US Air Force personnel (36). This aspect of an objective measurement of REE-based diet and nutrition counseling, may provide an additional tool in the treatment and management of overweight and obese adolescents, and needs to be explored further.

In conclusion, the results from the current study showed that MG underestimates traditional indirect calorimetry measured REE systematically in overweight and obese adolescents, and cannot be recommended for routine use. The Molnar equation of predicting REE provides similar estimates to measured REE by a standard indirect calorimeter in this vulnerable population, and can be recommended, when traditional indirect calorimeters are not available. The possible application of a portable device in the day-to-day practice of weight management interventions for obese adolescents, especially in the outpatient setting is quite attractive. Obesity management in adolescents should be individually tailored and several factors should be taken into account, including sex, age, the degree of obesity, individual health risks, psycho-behavioral, and metabolic characteristics. A handheld indirect calorimeter (MG) provides a practical alternative to traditional indirect calorimetry. Future studies are necessary to determine whether individually measured REE using handheld devices contributes to adolescent overweight/obese patient education, and improves effectiveness of weight loss recommendations.

\section{METHODS}

\section{Participants}

Forty-one children aged $12-18$ y who were overweight as defined by BMI between 15th and 85th percentile were recruited from the BC Children's Hospital outpatient clinics and by study advertisements in clinical areas of the hospital. Fifteen children with similar age and normal weight were recruited from the community to establish study day protocol, and trouble shoot problems with usage of the hand held indirect calorimeter. All potential participants interested in the study underwent a phone prescreening, during which time a research assistant answered questions, and explained the study objectives and procedures. Written informed consent was obtained from all participants and legal representative before study start. All research protocols were approved by the University of British Columbia/Children's and Women's Health Centre of British Columbia Research Ethics Board (UBC C\&W REB).

\section{Study Day Procedures}

The same trained technicians (P.W., G.M.) carried out all REE measurements in a quiet and temperature-controlled $\left(22^{\circ} \mathrm{C}\right)$ room in the Clinical Research and Evaluation Unit, BC Children's Hospital. 
Children arrived for the study after an overnight fast (10-12h), including abstention from all beverages except water. All children reported limited physical activity in the $8 \mathrm{~h}$ period prior to testing. Basic demographic information was collected using a questionnaire. Anthropometric measurements including body weight measured to the nearest $0.1 \mathrm{~kg}$ with an electronic scale, and height without shoes was measured to the nearest $0.1 \mathrm{~cm}$ with a stadiometer. Participants voided prior to arrival for the study. REE was determined using the hand-held indirect calorimeter (MG) and standard indirect calorimeter (VMax) on the same day for each participant in a random order.

REE Measurement Using Standard Indirect Calorimeter (VMax) Open-circuit indirect calorimetry (VMax Encore VE29n, Viasys Healthcare systems, Mississauga, ON, Canada) was performed using a clear ventilated canopy hood to measure oxygen consumption $\left(\mathrm{VO}_{2}\right)$ and carbon dioxide production $\left(\mathrm{VCO}_{2}\right)$ for $25 \mathrm{~min}$ (5). The VMax was calibrated daily using a certified calibration syringe for flow. Gas sensors for $\mathrm{CO}_{2}$ and $\mathrm{O}_{2}$ were calibrated prior to each measurement with standardized gases. Children remained in supine position, awake and motionless prior to and throughout the testing period to ensure valid and reproducible results (5). All children had their head slightly raised in order to minimize breathing irregularities, if any. The flow rate of room air drawn through the canopy was adjusted manually to maintain the fraction of $\mathrm{CO}_{2}$ expired at optimal range $(0.6-0.9 \%)$. Data from the first $5 \mathrm{~min}$ of the testing period were excluded to allow participant acclimatization (5). Steady state was defined as intervals of time (a minimum of $10 \mathrm{~min}$ ), during which average $\mathrm{VO}_{2}$ and $\mathrm{VCO}_{2}$ varied less than $10 \%$, and the respiratory quotient $\left(\mathrm{RQ}, \mathrm{VCO}_{2} / \mathrm{VO}_{2}\right)^{2}$ varied $\leq 5 \%$. The Vmax software, which utilizes the modified Weir equation, was used to compute REE (5).

\section{REE Measurement Using Portable Indirect Calorimeter (Medgem, MG)}

The hand-held indirect calorimeter used in this study was the MedGem (Microlife, Golden, CO). The device consists of an oxygen sensor and flow sensor to measure $\mathrm{VO} 2$, and REE is estimated using the Weir equation (5) under the assumption of a constant RQ (11). All participants rested in a relaxed seated position for $10 \mathrm{~min}$ prior to measurements. Participants stayed awake, upright, and motionless throughout the testing period $(8-12 \mathrm{~min})$. The device was calibrated placing it on a flat surface before each measurement, as per manufacturer instructions. The nose clip was placed on the participant's nose to close the nostrils and prevent breathing through the nose and the mouthpiece was positioned in the mouth. Care was taken to ensure a firm seal around the mouthpiece. Each child was permitted to test the mouthpiece prior to testing to ensure comfort of nose clip and to check for air leaks. Blanket rolls were used to prop up the arms of the children to minimize discomfort or unnecessary movements. Children held the MedGem device in place until it beeped, indicating end of measurement.

\section{REE Prediction Using Equations}

At BC Children's Hospital (BCCH), The Harris Benedict equation (32), $\mathrm{WHO} / \mathrm{FAO} / \mathrm{UNU}$ equation (33), and the Molnar equation (26) (not in order of preference) are the three most commonly used to predict energy needs in the overweight/obese population. Hence, for the purpose of this study, REE was calculated using the above predictive equations, to compare against the measured REE using MG and VMax.

\section{Body Composition Measurement}

All children had their body composition analyzed using bioelectrical impedance analysis immediately following REE measurements with the standard indirect calorimeter, while still lying in a supine position. This allowed for stabilization of fluid volume. Children were positioned with limbs apart. Two electrodes were placed on the hand and foot as previously described (37). Body composition was determined using a bioelectrical impedance analyzer (Quantum IV, RJL Systems, Detroit, MI) at a frequency of $50 \mathrm{kHz}$ with a constant current of $0.8 \mathrm{~mA}$ to obtain impedance (38). Three measurements on each child, not to vary by more than $1 \Omega$, were taken (37). Total body water was estimated using the resistance index $\left(\mathrm{RI}=\right.$ height $^{2} /$ resistance $)$ and age-/sex-specific equations $(39,40)$ were used to calculate fat mass and fat-free mass.

\section{Statistical Analysis}

Results are represented as mean \pm SD. Paired student $t$-test was used to assess systematic differences between REE measured by VMax and MG. Pearson's correlation analysis was used to detect linear agreement between REE measured using VMax and MG. Since correlation between measures does not provide information about the differences between the two measures, Bland-Altman analysis (41-44) was used to assess the degree of agreement, mean bias and proportional bias between the two indirect calorimeter assessments of REE. Mean bias determines the quantitative difference between the two measurements, and the proportional bias determines whether the magnitude of the measurement affects the quantitative difference. In order to interpret Bland-Altman analysis, a $\pm 250 \mathrm{kcal} / \mathrm{d}$ in REE measured by the two techniques was set a priori as a clinically significant difference. This is based on the calculation that a $250 \mathrm{kcal} / \mathrm{d}$ change in energy intake would lead to a half-pound weight loss in a week, as previously applied by Curtin et al. (28), which is in line with recommendations by the American Academy of Pediatrics for treatment of and overweight and obese adolescents $(3,29)$.

Data were analyzed using SPSS version 17 (SPSS, Chicago, IL) and Bland-Altman plots were constructed using Med-Calc version 9.3.1 (MedCalc Software, Ostend, Belgium).

\section{ACKNOWLEDGMENTS}

We would like to thank Jennifer Kwan for help with study coordination. The study was registered under Clinical Trials.Gov (NCT01776944).

\section{STATEMENT OF FINANCIAL SUPPORT}

This study was supported in part by a Research Institute, BC Children's Hospital (Vancouver, Canada) Establishment Award (RE).

Disclosure: Authors have no conflicts of interest to disclose

\section{REFERENCES}

1. Statistics Canada. Canadian Health Measures Survey, 2009-2011. (http:// www.statcan.gc.ca/pub/82-003-x/2012003/article/11706/tbl/tbl2-eng. htm).

2. Lau DC, Douketis JD, Morrison KM, Hramiak IM, Sharma AM, Ur E; Obesity Canada Clinical Practice Guidelines Expert Panel. 2006 Canadian clinical practice guidelines on the management and prevention of obesity in adults and children [summary]. CMAJ 2007;176:S1-13.

3. The Academy of Nutrition and Dietetics. Evidence Analysis Library. Pediatric Weight Management Guidelines 2007. (https://www.andeal.org/topic. cfm?cat=2721).

4. Holdy KE. Monitoring energy metabolism with indirect calorimetry: instruments, interpretation, and clinical application. Nutr Clin Pract 2004;19:447-54.

5. da Rocha EE, Alves VG, da Fonseca RB. Indirect calorimetry: methodology, instruments and clinical application. Curr Opin Clin Nutr Metab Care 2006;9:247-56.

6. Branson RD, Johannigman JA. The measurement of energy expenditure. Nutr Clin Pract 2004;19:622-36.

7. Wells JC, Fuller NJ. Precision and accuracy in a metabolic monitor for indirect calorimetry. Eur J Clin Nutr 1998;52:536-40.

8. Elia M, Livesey G. Theory and validity of indirect calorimetry during net lipid synthesis. Am J Clin Nutr 1988;47:591-607.

9. Goran MI, Nagy TR. Effect of the pre-testing environment on measurement of metabolic rate in children. Int J Obes Relat Metab Disord 1996;20:83-7.

10. Compher C, Frankenfield D, Keim N, Roth-Yousey L; Evidence Analysis Working Group. Best practice methods to apply to measurement of resting metabolic rate in adults: a systematic review. J Am Diet Assoc 2006;106:881-903.

11. McDoniel, SO. A systematic review on use of a handheld indirect calorimeter to assess energy needs in adults and children. International Journal of Sport Nutrition and Exercise Metabolism 2007;7:491-500.

12. Madden AM, Parker LJ, Amirabdollahian F. Accuracy and preference of measuring resting energy expenditure using a handheld calorimeter in healthy adults. J Hum Nutr Diet 2013;26:587-95. 
13. Fares S, Miller MD, Masters S, Crotty M. Measuring energy expenditure in community-dwelling older adults: are portable methods valid and acceptable? J Am Diet Assoc 2008;108:544-8.

14. St-Onge M, Rubiano F, Jones A, Heymsfield S. A new handheld indirect calorimeter to measure postprandial energy expenditure. Int J Obes Relat Metab Disord 2004;28:1479-84.

15. Robins K, Stankorb SM, Salgueiro M. Energy expenditure in acute posttraumatic amputation: comparison of four methods for assessment. Nutr Clin Pract 2013;28:758-65.

16. Glass C, Hipskind P, Cole D, Lopez R, Dasarathy S. Handheld calorimeter is a valid instrument to quantify resting energy expenditure in hospitalized cirrhotic patients: a prospective study. Nutr Clin Pract 2012;27:677-88.

17. Compher C, Hise M, Sternberg A, Kinosian BP. Comparison between Medgem and Deltatrac resting metabolic rate measurements. Eur J Clin Nutr 2005;59:1136-41.

18. Hlynsky J, Birmingham CL, Johnston M, Gritzner S. The agreement between the MedGem indirect calorimeter and a standard indirect calorimeter in anorexia nervosa. Eat Weight Disord 2005;10:e83-7.

19. Reeves MM, Capra S, Bauer J, Davies PS, Battistutta D. Clinical accuracy of the MedGem indirect calorimeter for measuring resting energy expenditure in cancer patients. Eur J Clin Nutr 2005;59:603-10.

20. Anderson EJ, Sylvia LG, Lynch M, Sonnenberg L, Lee H, Nathan DM. Comparison of energy assessment methods in overweight individuals. J Acad Nutr Diet 2014;114:273-8.

21. Cooper JA, Watras AC, O’Brien MJ, et al. Assessing validity and reliability of resting metabolic rate in six gas analysis systems. J Am Diet Assoc 2009;109:128-32.

22. Dobratz JR, Sibley SD, Beckman TR, et al. Predicting energy expenditure in extremely obese women. JPEN J Parenter Enteral Nutr 2007;31:217-27.

23. Fields DA, Kearney JT, Copeland KC. MedGem hand-held indirect calorimeter is valid for resting energy expenditure measurement in healthy children. Obesity (Silver Spring) 2006;14:1755-61.

24. Nieman DC, Austin MD, Chilcote SM, Benezra L. Validation of a new handheld device for measuring resting metabolic rate and oxygen consumption in children. Int J Sport Nutr Exerc Metab 2005;15:186-94.

25. Patt PL, Agena SM, Vogel LC, Foley S, Anderson CJ. Estimation of resting energy expenditure in children with spinal cord injuries. J Spinal Cord Med 2007;30 Suppl 1:S83-7.

26. Hofsteenge GH, Chinapaw MJ, Delemarre-van de Waal HA, Weijs PJ. Validation of predictive equations for resting energy expenditure in obese adolescents. Am J Clin Nutr 2010;91:1244-54.

27. Molnár D, Jeges S, Erhardt E, Schutz Y. Measured and predicted resting metabolic rate in obese and nonobese adolescents. J Pediatr 1995;127: $571-7$.
28. Curtin C, Bandini LG, Must A, et al. Parent support improves weight loss in adolescents and young adults with Down syndrome. J Pediatr 2013;163:1402-8.e1.

29. Barlow SE; Expert Committee. Expert committee recommendations regarding the prevention, assessment, and treatment of child and adolescent overweight and obesity: summary report. Pediatrics 2007;120 Suppl 4:S164-92.

30. Heshka S, Feld K, Yang MU, Allison DB, Heymsfield SB. Resting energy expenditure in the obese: a cross-validation and comparison of prediction equations. J Am Diet Assoc 1993;93:1031-6.

31. Breen HB, Ireton-Jones CS. Predicting energy needs in obese patients. Nutr Clin Pract 2004;19:284-9.

32. Harris JA, Benedict FG. A biometric study of human basal metabolism. Proc Natl Acad Sci USA 1918;4:370-3.

33. Energy and protein requirements. Report of a joint $\mathrm{FAO} / \mathrm{WHO} / \mathrm{UNU}$ expert consultation. World Health Organ Tech Rep Ser 1985;724:1-206.

34. Hill JO. Can a small-changes approach help address the obesity epidemic? A report of the Joint Task Force of the American Society for Nutrition, Institute of Food Technologists, and International Food Information Council. Am J Clin Nutr 2009;89:477-84.

35. Hills AP, Byrne NM, Lindstrom R, Hill JO. 'Small changes' to diet and physical activity behaviors for weight management. Obes Facts 2013;6:228-38.

36. McDoniel SO, Nelson HA, Thomson CA. Employing RMR technology in a 90-day weight control program. Obes Facts 2008;1:298-304.

37. Kushner RF, Schoeller DA. Estimation of total body water by bioelectrical impedance analysis. Am J Clin Nutr 1986;44:417-24.

38. Jaffrin MY. Body composition determination by bioimpedance: an update. Curr Opin Clin Nutr Metab Care 2009;12:482-6.

39. Schaefer F, Georgi M, Zieger A, Schärer K. Usefulness of bioelectric impedance and skinfold measurements in predicting fat-free mass derived from total body potassium in children. Pediatr Res 1994;35:617-24.

40. Wabitsch M, Braun U, Heinze E, et al. Body composition in 5-18-y-old obese children and adolescents before and after weight reduction as assessed by deuterium dilution and bioelectrical impedance analysis. Am J Clin Nutr 1996;64:1-6.

41. Bland JM, Altman DG. Statistical methods for assessing agreement between two methods of clinical measurement. Lancet 1986;1:307-10.

42. Ludbrook J. Statistical techniques for comparing measurers and methods of measurement: a critical review. Clin Exp Pharmacol Physiol 2002;29: 527-36.

43. Hopkins WG. Bias in Bland-Altman but not regression validity analyses. Sportsscience 2004;8:42-46.

44. Batterham AM. Commentary on bias in Bland-Altman but not regression validity analyses. Sportsscience 2004;8:47-49. 